University of Nebraska - Lincoln

DigitalCommons@University of Nebraska - Lincoln

Faculty Publications in Computer \& Electronics Electrical \& Computer Engineering, Department Engineering (to 2015)

2011

\title{
A Study On Energy Efficient Multi-Tier Multi-Hop Wireless Sensor Networks For Freight-Train Monitoring
}

\author{
Puttipong Mahasukhon \\ University of Nebraska-Lincoln, pmahasukhon@huskers.unl.edu \\ Hamid Sharif \\ University of Nebraska-Lincoln, hsharif@unl.edu \\ Michael Hempel \\ University of Nebraska-Lincoln, mhempel2@unl.edu \\ Ting Zhou \\ University of Nebraska-Lincoln, tzhou@unInotes.unl.edu \\ Tao Ma \\ University of Nebraska Lincoln, tma@unInotes.unl.edu \\ See next page for additional authors
}

Follow this and additional works at: https://digitalcommons.unl.edu/computerelectronicfacpub

Part of the Computer Engineering Commons

\begin{abstract}
Mahasukhon, Puttipong; Sharif, Hamid; Hempel, Michael; Zhou, Ting; Ma, Tao; and Shrestha, Pradhumna, "A Study On Energy Efficient Multi-Tier Multi-Hop Wireless Sensor Networks For Freight-Train Monitoring" (2011). Faculty Publications in Computer \& Electronics Engineering (to 2015). 100.

https://digitalcommons.unl.edu/computerelectronicfacpub/100

This Article is brought to you for free and open access by the Electrical \& Computer Engineering, Department of at DigitalCommons@University of Nebraska - Lincoln. It has been accepted for inclusion in Faculty Publications in Computer \& Electronics Engineering (to 2015) by an authorized administrator of DigitalCommons@University of Nebraska - Lincoln.
\end{abstract}




\section{Authors}

Puttipong Mahasukhon, Hamid Sharif, Michael Hempel, Ting Zhou, Tao Ma, and Pradhumna Shrestha 


\title{
A STUDY ON ENERGY EFFICIENT MULTI-TIER MULTI-HOP WIRELESS SENSOR NETWORKS FOR FREIGHT-TRAIN MONITORING
}

\author{
Puttipong Mahasukhon, Hamid Sharif, Michael Hempel, Ting Zhou, Tao Ma, and Pradhumna Lal Shrestha \\ Department of Computer and Electronics Engineering \\ University of Nebraska-Lincoln \\ Omaha, NE, USA \\ \{pmahasukhon, hsharif, mhempel, tzhou, tma, plshrestha\}@unlnotes.unl.edu
}

\begin{abstract}
The North American freight railroad industry is trying to leverage wireless sensor networks (WSN) onboard railcars for advanced monitoring and alerting. In railroad environments, freight train WSNs exhibit a linear chain-like topology of significant length. Thus, existing wireless technologies such as the IEEE 802.15.4 communication protocol, based on a star topology, are unable to provide reliable service. The end-to-end communication between nodes generally relies on individual nodes communicating with their respective neighbors to carry the information over multiple hops and deliver it to the preferred destination. The routing performance and reliability significantly degrades with increasing number of hops. We proposed a multitier multi-hop network which is designed to overcome these issues in large-scale multi-hop WSNs in railroad environments. This approach has significant advantages, such as more data bandwidth, higher reliability, and lower energy consumption. Our analytical results show that the proposed multi-tier communication approach spends energy more efficiently and utilizes less resource than the traditional chain topology onboard freight trains.
\end{abstract}

Keywords-IEEE 802.15.4; large-scale multi-hop network; wireless sensor networks; wireless personal area networks; energy

\section{INTRODUCTION}

In North America's freight railroad industry, a number of efforts are underway to improve the safety and security of freight transportation, such as Positive Train Control (PTC) and efforts for real-time train consist health monitoring. The freight railroad companies see Wireless Sensor Networks (WSNs) as a potential technology for real-time status monitoring of each individual railcar and reporting changes in status to the train operator and dispatch center for alerting purposes. Such a system could monitor a wide variety of conditions, such as wheel imbalances, problems with the brake system in a railcar, the failure of a refrigeration unit when transporting perishable goods, etc. Freight trains can consist of a very large number of railcars. In each onboard railcar, individual WSN nodes are tasked with data collection and reporting.

The standard IEEE 802.15.4 [1] specifies low-rate wireless

This research is supported by the Federal Railroad Administration (FRA) under grant number 25-1105. The authors are with the Advanced Telecommunications Engineering Laboratory (www.TEL.unl.edu). personal area networks (LR-WPAN) supporting small, lowcost, but power-efficient devices operating on battery power. The IEEE 802.15.4 protocol is widely adopted in a variety of embedded applications, such as home automation [2], industrial sensing and control, environment monitoring and sensing, and wireless peripheral equipments. The IEEE 802.15.4, also known as ZigBee, is envisioned by the railroads to provide the interconnectivity for these railcar sensor nodes. However, even though the IEEE 802.15.4 protocol supports multi-hop communications, it was originally designed for star topologies and is therefore not optimized when used in large-scale multihop chain-topology networks.

Research related to the performance of IEEE 802.15.4 in a star topology has been reported in [3]. The authors of [4] identified the performance bottlenecks in the MAC layer of 802.15.4. In many applications that utilize a chain topology the performance problems are intensified due to IEEE 802.15.4's limited communication range. Unlike IEEE 802.11, an RTS/CTS handshake is not included in the IEEE 802.15.4 protocol. Therefore, IEEE 802.15.4 does not handle the hidden terminal problem appropriately in multi-hop environments. From these problems associated with IEEE 802.15.4 it becomes obvious that improvements are required in order to develop a reliable and robust solution for chain-topology multihop wireless sensor networks. Furthermore, the route discovery becomes significantly more difficult and takes longer time to complete as the number of hops increases. In [5], the authors reported the maximum IEEE 802.15.4 multi-hop throughput. According to their findings, the maximum throughput is decreased from $250 \mathrm{kbps}$ to $40 \mathrm{kbps}$ as the number of hops increases from 1 hop to 5 hops.

In this paper, we focus on the energy efficiency of largescale multi-hop WSNs onboard freight trains. A typical size in our consideration is around 100 railcars. If there is one sensor deployed per freight car, the end-to-end network length is 99 hops. This hop count far exceeds the original design capabilities of IEEE 802.15.4. We therefore propose a multitier multi-hop network approach as an effective solution to the problems associated with IEEE 802.15.4 in such an environment. Detailed performance evaluations and analyses provide a guideline for practical implementations of WSNs onboard freight trains. 


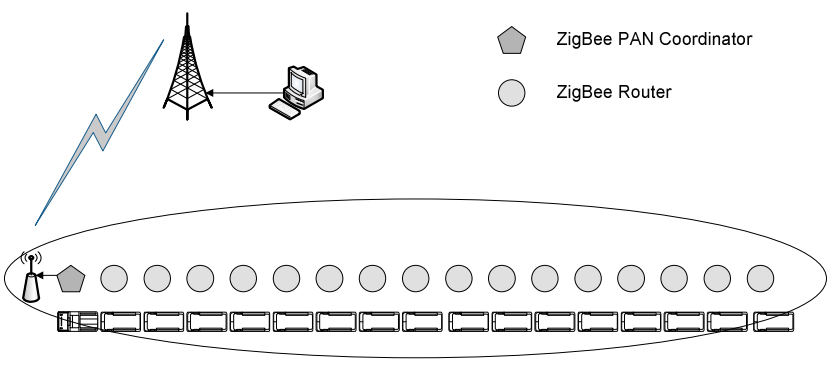

Fig. 1. A large-scale multi-hop sensor network in railroad application with one ZigBee device on each freight car

The paper is organized as follows. Section II provides an overview of the IEEE 802.15.4 standard and multi-hop problems. Section III presents our proposed solution for largescale multi-hop sensor networks. Our simulation results are given in Section IV. Finally, Section V concludes this paper.

\section{BACKGROUND}

\section{A. IEEE 802.15.4 Standard}

The IEEE 802.15.4 [1] standard specifies the physical layer and the MAC sub-layer for Low-Rate Wireless Personal Area Networks (LR-WPANs). IEEE 802.15.4 offers three operational frequency bands: $2.4 \mathrm{GHz}, 915 \mathrm{MHz}$, and 868 MHz. There is a single channel allocated between 868 and 868.6 MHz, 10 channels between 902 and $928 \mathrm{MHz}$, and 16 channels between 2.4 and $2.4835 \mathrm{GHz}$. The maximum data transfer rates are $250 \mathrm{kbps}$ at $2.4 \mathrm{GHz}, 40 \mathrm{kbps}$ at $915 \mathrm{MHz}$ and $20 \mathrm{kbps}$ at $868 \mathrm{MHz}$. In this paper, we consider the physical layer operating in the $2.4 \mathrm{GHz}$ frequency band, which is supported by the MICAz motes [6] from Crossbow Technology.

In the IEEE 802.15.4 standard, there are two types of devices: Full Function Devices (FFDs) and Reduced Function Devices (RFDs). The FFD has full 802.15.4 functionality, including network routing functionality, whereas the RFD provides limited functionality supporting only simple applications such as a remote-controlled switch or data collection sensor to reduce cost and complexity. An IEEE 802.15.4 network consists of at least one FFD operating as the PAN coordinator. Both device types can be interconnected to form either a simple star or a self-configuring peer-to-peer topology. Peer-to-peer topologies are suitable for industrial and commercial applications, where efficient self-configurability and large coverage are important, but its disadvantage is the increased network latency due to message relaying.

\section{B. Multi-hop Network}

Multi-hop wireless networks are an ideal technology to establish an instant communication infrastructure for railroad applications. However, as the size of a multi-hop network grows, the performance tends to decrease. Key causes of such degradation include the resulting excessive control traffic overhead required to build and maintain accurate routing tables. Thus, it is difficult to guarantee performance on a path with many wireless hops, which is particularly critical for the support of real time applications.

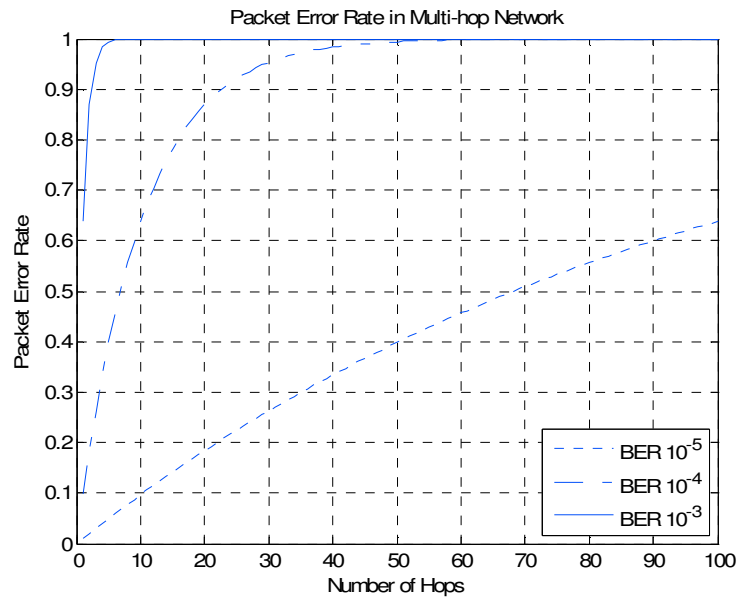

Fig. 2. Packet error rate at specific number of hops in the chain multi-hop scenario when the packet size is 127 bytes.

In addition to the routing issue, the possibility of successful packet delivery is lower as the number of hops increases. This is due to received bit/packet error in wireless communication which can be expressed as follow:

$$
\begin{gathered}
P E R=1-(1-B E R)^{\text {number_bits }}, \\
P E R_{\text {Multihop }}=1-(1-P E R)^{\text {number_hops }} .
\end{gathered}
$$

The $B E R$ is the bit error rate, which is the number of bit errors divided by the total number of transferred bits during a studied time interval. The packet error rate $(P E R)$ is the number of incorrectly transferred data packets divided by the number of transferred packets. Fig. 2 shows the packet error rate at specific number of hops in the chain multi-hop scenario when the packet size is 127 bytes. If the link quality is good for entire network, let say BER of $10^{-5}$, the successful packet delivery is still less than $40 \%$. It is certain that retransmission plays a big part in large-scale multi-hop network. In the railroad environment which is considered as a harsh environment, it is very difficult to achieve that good link quality.

\section{Multi-TIER Multi-HOP NetwORK}

Due to the poor performance of wireless sensor networks where a large number of hops are involved, a multi-tier multihop network approach was proposed to significantly reduce the routing overhead, which is identified as the primary problem associated with traditional multi-hop approaches in freight train WSNs. The idea behind the multi-tier multi-hop network is to divide a very long chain-topology network into several short segments and each segment is connected to its neighboring segments using a longer-distance communication protocol, such as $\mathrm{Wi}-\mathrm{Fi}$, to greatly reduce the number of required hops for route discovery and data forwarding. Fig. 3 illustrates the multi-tier multi-hop approach. In the upper-tier interconnect, Wi-Fi technology is our preferred choice because it provides 


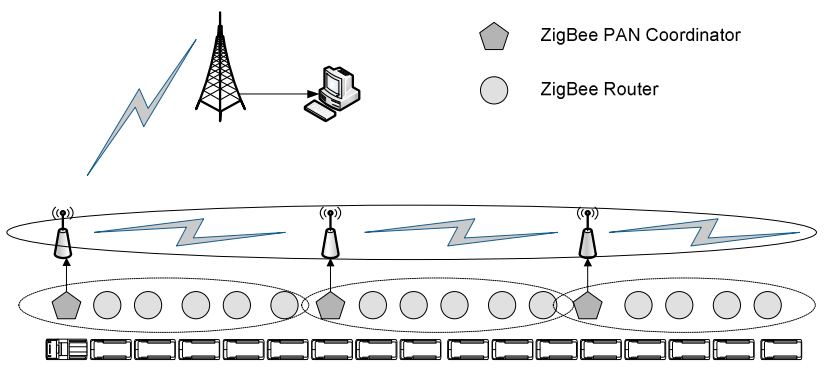

Fig. 3. A multi-tier multi-hop network with one ZigBee device on each freight car

longer range, higher bandwidth, and faster data transfer rates than ZigBee. Moreover, at the current-state-of-art, the cost of ultra-low powered Wi-Fi chipset has moved close to the cost of ZigBee chipsets. The Wi-Fi interface of each node forms an upper-tier multi-hop WLAN network. On the lower tier multihop networks, a very long chain multi-hop WPAN network can now be divided into several short-chain multi-hop WPAN networks. Each WPAN has its own PAN coordinator and works independently of the other WPANs. Each ZigBee device now needs only a few hops to reach its local PAN coordinator, which is the node designated to also act as the gateway to the WLAN network. If there is a sensor node failure, this will be of significance only to the WPAN containing the node, not the entire network and thus isolating failure points and increasing the network robustness.

In this paper, we consistently use 100 as the typical number of railcars. If there is one sensor deployed per freight car, the maximum end-to-end network length is 99 hops. In the multitier multi-hop network, a 100-node WPAN network using the traditional single-tier approach can be subdivided into 10 individual WPANs using our approach. With this approach, we can reduce the maximum hop count from 99 hops to less than 20 hops (9 WPAN hops and 9 Wi-Fi hops). This number is far more attractive and reasonable, particularly when considering real world implementations.

The advantages of this approach, in addition to the significant reduction in the number of hops, are the higher bandwidth on the upper tier, the increased flexibility, and the greater reliability. Consequently, the overall system throughput is increased as we reported in [7].

\section{ANALYTICAL RESULTS}

For the performance evaluation of our proposed approach, we setup a scenario with 100 freight cars, each car with one ZigBee router. In multi-tier multi-hop analysis, we divide this large WPAN into ten individual WPANs interconnected by one higher-tier Wi-Fi WLAN. One WPAN therefore consists of one PAN coordinator and nine ZigBee routers. Each PAN coordinator has co-located ZigBee and Wi-Fi chipsets. With this approach the maximum number of hops in a WPAN decreases from 99 to 9 . In addition, the Wi-Fi higher-tier interconnect encompasses at most 9 hops. In the past, we have setup and tested a similar Wi-Fi chain topology of $8 \mathrm{Wi}-\mathrm{Fi}$ hops in our 3.5-mile test bed without any difficulties. This test bed was established on track operated by Burlington Northern

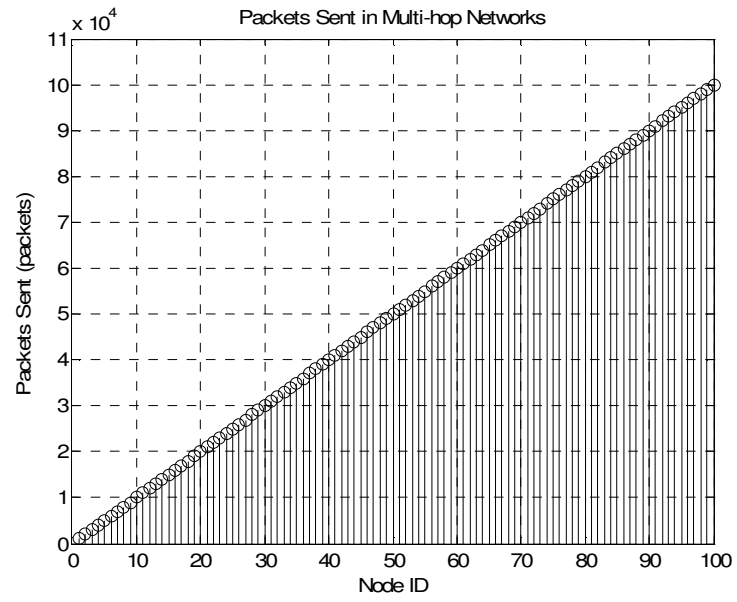

Fig. 4. Expected number of data packets sent at each hop in the multi-hop scenario when the duration is 1,000 seconds and the CBR traffic rate of each node is one packet per second.

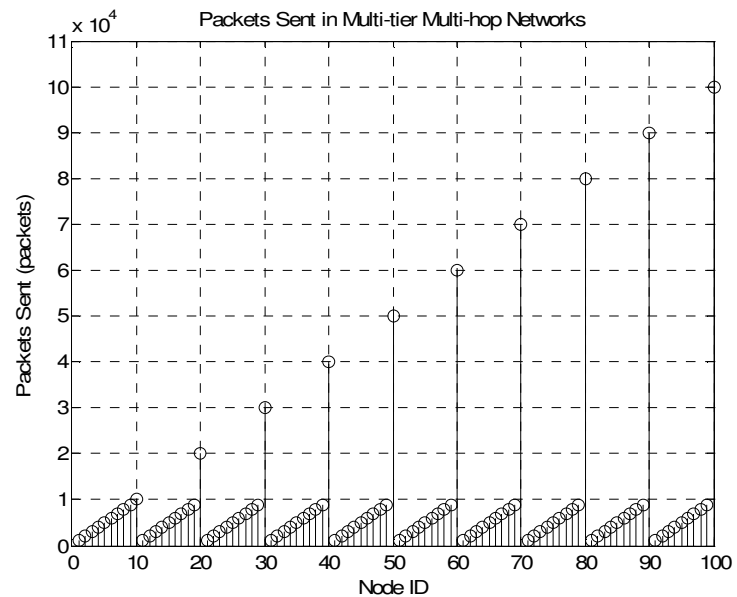

Fig. 5. Expected number of data packets sent at each hop in the multi-tier multi-hop scenario when the duration is 1,000 seconds and the CBR traffic rate of each node is one packet per second.

Santa Fe (BNSF) in Crete, Nebraska to investigate the impact of mobility on end-to-end $\mathrm{Wi}$-Fi performance. A detailed report on this test bed is provided in [8].

After all nodes successfully completed synchronization, every sensor transmits as many data packets as possible to the sink node, which is designated as node 100. Each node has a constant bit rate (CBR) traffic source generating at a rate of one packet per second. The packet size is set to 70 bytes. The intermediate nodes are also responsible for forwarding packets to their neighbors. Similarly, the Wi-Fi higher-tier interconnect effectively aggregates more and more data packets the closer it is to the sink node. In the multi-hop scenario, nodes 1 , $2, \ldots, 99$ are the FFD devices, which are capable of forwarding packets, while node 100 is the PAN coordinator, which generates beacon frames for its own WPAN network. In the multi-tier multi-hop scenario, nodes $10,20, \ldots, 100$ are the PAN coordinators and the rest are the FFD devices. 


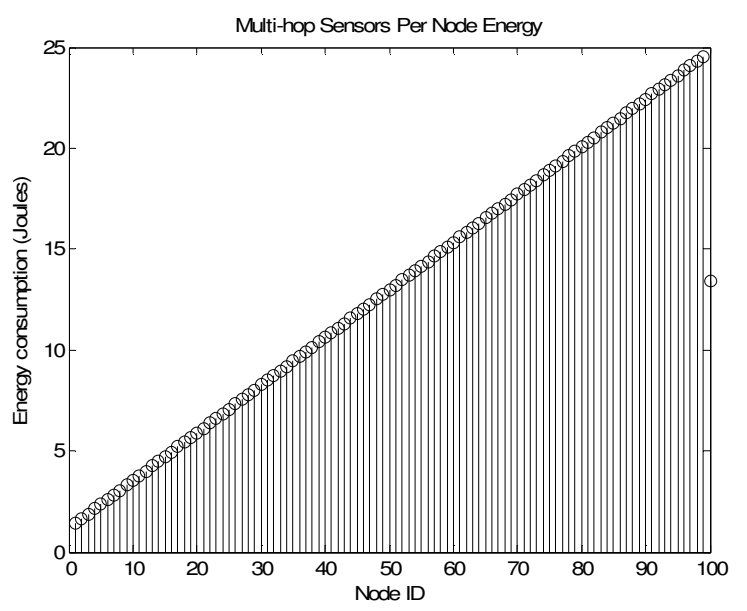

Fig. 6. Energy consumption of each node in the multi-hop scenario when the duration is 1,000 seconds and the CBR traffic rate of each node is one packet per second.

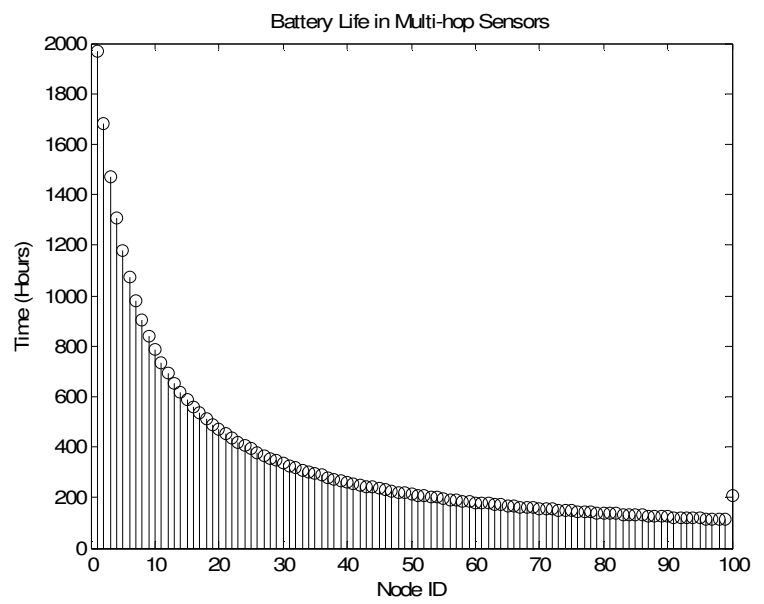

Fig. 7. Lifetime of each node in the multi-hop scenario when the CBR traffic rate of each node is one packet per second.

For the regular multi-hop network, the maximum number of packets per hop is limited by the 2-hop shared-channel saturation throughput of $125 \mathrm{kbps}$, since each node has two neighbors in its communication range. Thus, the maximum number of packets at the destination is 223,214 packets during the 1000-second observation, with the destination node theoretically receiving 2,232 packets from each sensor node. In the multi-tier multi-hop network, the maximum number of packets per hop in each WPAN segment is also limited to 223,214 packets. However, each PAN coordinator is able to handle a significantly larger number of packets due to its integrated Wi-Fi network access. In this environment, the total number of packets at the destination can be increased to $6,696,428$ packets, which is approximately 30 times larger compared to the regular multi-hop network and scales favorably with the number of segmented WPANs.

Fig. 4 and Fig. 5 illustrate the number of packets sent in the regular multi-hop scenario and the multi-tier multi-hop scenario, respectively. Both of them represent the amount of

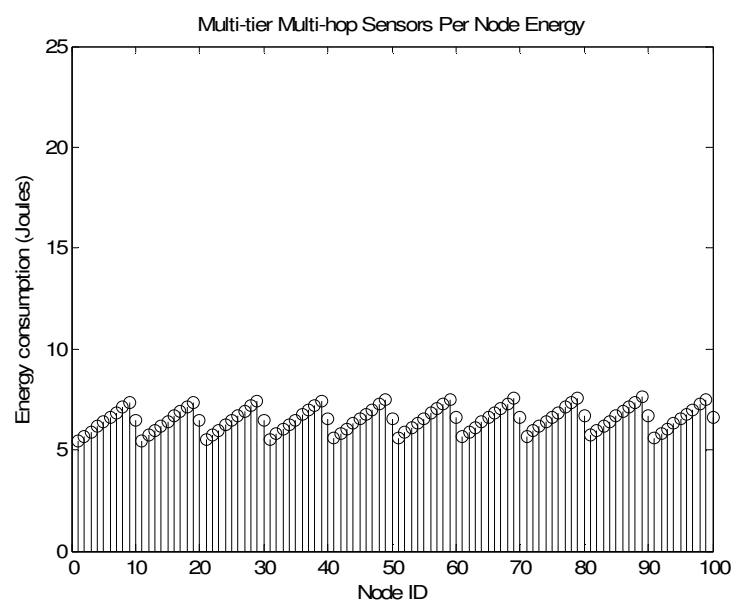

Fig. 8. Energy consumption of each node in the multi-tier multi-hop scenario when the duration is 1,000 seconds and the CBR traffic rate of each node is one packet per second.

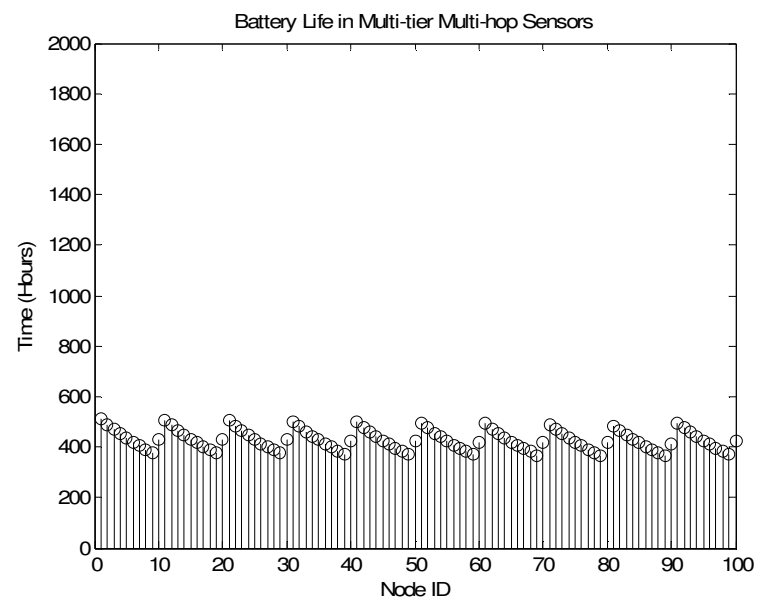

Fig. 9. Lifetime of each node in the multi-tier multi-hop scenario when the CBR traffic rate of each node is one packet per second.

packets each node generates and forwards during 1,000 seconds. As can be seen from these graphs, the number of packets that need to be exchanged between nodes in multi-hop is much higher than the corresponding number in its multi-tier counterpart. The worst case is when the node 1 sends a packet to the node 100 . It has to be sent through node 2 . The packet is then forwarded 98 more times (by node $2,3, \ldots$, and 99) in the multi-hop scenario, whereas in the proposed multi-tier multihop scenario it is forwarded only more 8 times using nodes 2 , $3, \ldots$, and 9 in the sensor lower-tier and another 9 times by node $10,20, \ldots$, and 90 in the Wi-Fi upper-tier. Therefore, the maximum possible number of hops is obviously reduced from 99 hops in the multi-hop network to 18 hops in the multi-tier multi-hop network.

From the results, we can see that the nodes which are closer to the destination spend most of the time forwarding packets. This makes the multi-hop network less efficient with longer the topology chain becomes longer. In addition, the energy stored in those sensor nodes depletes rapidly because they have to be 
TABLE I. WIRELESS SENSOR NODE POWER CONSUMPTION

\begin{tabular}{|l|l|}
\hline \multicolumn{1}{|c|}{ Sensor } & \multicolumn{1}{c|}{ Power Consumption } \\
\hline RxPower & $56 \mathrm{~mW}$ \\
\hline TxPower & $52 \mathrm{~mW}$ \\
\hline IdlePower & $1.3 \mathrm{~mW}$ \\
\hline SleepPower & $48 \mathrm{uW}$ \\
\hline
\end{tabular}

TABLE II. LOW-POWER WI-Fi NODE POWER CONSUMPTION

\begin{tabular}{|l|l|}
\hline \multicolumn{1}{|c|}{ Wi-Fi } & \multicolumn{1}{c|}{ Power Consumption } \\
\hline RxPower & $40 \mathrm{~mW}$ \\
\hline TxPower & $360 \mathrm{~mW}$ \\
\hline IdlePower & $40 \mathrm{~mW}$ \\
\hline SleepPower & $36 \mathrm{uW}$ \\
\hline
\end{tabular}

awake all the time to keep forwarding packets and do not get a chance to go to sleep mode for energy saving. In an energy constrained system like WSN, this energy concern is very critical. Therefore, we have analyzed energy usage of each node in both traditional multi-hop and proposed multi-tier multi-hop scenarios. We adopted wireless sensor parameters from Texas Instrument's CC2420 chipset specification [9] as shown in Table I. Fig. 6 demonstrates the energy consumption of each node in the multi-hop scenario during a 1000-second simulation where the CBR traffic rate of each node is one packet per second. The energy usage in this scenario can be up to 25 Joules and the range of the nodes' energy usage is considerably large. This signifies an unfair energy usage in this scenario. Typical size AA NiMH batteries have a capacity of about 10,000 Joules. For our analysis, we used this number to determine how long the battery of each sensor node can last. Fig. 7 demonstrates the battery life of each node in the multihop scenario.

Table II shows the power consumption of ultra-low-power WiFly RN-111B module from Roving Networks [10]. We utilized these numbers for the Wi-Fi interfaces. Fig. 8 demonstrates energy consumption of each node in the multitier multi-hop scenario during a 1000-second simulation for the same CBR traffic rate of one packet per second. The energy consumption is only ranging from 5 to 8 Joules in this scenario. Fig. 9 illustrates the battery life of each node in the multi-tier multi-hop scenario. Table III summarizes the node lifetime in both scenarios. The most important number is the minimum node lifetime because it also reflects the network lifetime. According to our simulation configuration, the proposed multitier multi-hop network can significantly improve the network lifetime from 113.1 hours to 364.92 hours.

\section{CONCLUSION}

This paper provides an analysis of multi-hop WSN communication onboard freight trains using traditional singletier IEEE 802.15.4 communication and assuming a chain-like topology. We have shown the performance degradation when the size of this multi-hop network, and thus the chain length,
TABLE III. NODE LIFETIME

\begin{tabular}{|l|c|c|}
\hline \multirow{2}{*}{\multicolumn{1}{|c|}{ Network Type }} & \multicolumn{2}{|c|}{ Node Lifetime (hours) } \\
\cline { 2 - 3 } & Min & Max \\
\hline Multi-hop Network & 113.1 & $1,965.1$ \\
\hline Multi-tier Multi-hop Network & 364.92 & 510.03 \\
\hline
\end{tabular}

grows. We have also shown that the proposed multi-tier multihop network approach for freight-train WSNs can improve scalability by reducing the number of transmission hops required to reach the destination with the help of a multi-tier network architecture. Compared with the traditional single-tier communication, our analytical study indicates that the use of the proposed multi-tier counterpart can clearly achieve lower energy consumption and less network resource utilization, which is an attractive solution in large-scale multi-hop networks. As a result, the node lifetime of each individual sensor also approaches a more uniform distribution, which increases the overall network lifetime.

\section{ACKNOWLEDGMENT}

This study is being conducted at the University of Nebraska-Lincoln by the Advanced Telecommunications Engineering Laboratory (www.TEL.unl.edu) researchers, with support from the US Federal Railroad Administration (FRA) under the direction of John Punwani and Shahram Mehrvarzi. This study has also been supported by Union Pacific Railroad and the Association of American Railroads (AAR)..

\section{REFERENCES}

[1] IEEE 802.15.4, "Part 15.4: Wireless Medium Access Control (MAC) and Physical Layer (PHY) Specifications for Low Rate Wireless Personal Area Networks (LR WPANs)", IEEE SA Standards Board, 2003.

[2] E. Callaway, P. Gorday, L. Hester et al, "Home Networking with IEEE 802.15.4: A Developing Standard for Low-Rate Wireless Personal Area Networks", IEEE Communications Magazine, 2002, 40(8): 70 -77.

[3] J. Zheng and M. J. Lee, "Will IEEE 802.15.4 make ubiquitous networking a reality? : A discussion on a potential low power low bit rate standard", IEEE Communications Magazine, 2004, 42:140-146.

[4] J. Misic, S. Shafi, and V. B. Misic, "Avoiding the Bottlenecks in the MAC Layer in 802.15.4 Low-Rate WPAN", in Proc. of 11th International Conference on Parallel and Distributed Systems Workshops, 2005, pp. 363-367.

[5] F. Osterlind and A. Dunkels, "Approaching the Maximum 802.15.4 Multi-hop Throughput," SICS Technical Report T2008:05, 2008.

[6] MICAz Datasheet; Available at http://www.xbow.com/.

[7] P. Mahasukhon, H. Sharif, M. Hempel, T. Zhou, W. Wang, and T. Ma, "Multi-tier multi-hop routing in large-scale wireless sensor networks for freight-train monitoring," in Proc. of ITS World Congress'10, Busan, Korea.

[8] M. Hempel, H. Sharif, T. Zhou, and P. Mahasukhon, "A wireless test bed for mobile 802.11 and beyond," in Proc. of IWCMC'06, Vancouver, Canada, July 2006, pp. 1003-1008.

[9] Texas Instument CC2420 Datasheet; Available at http://focus.ti.com/docs/prod/folders/print/cc2420.html.

[10] Roving Networks WiFly RN-111B Module; Avalable at http://www.rovingnetworks.com/rn-111.php. 